\title{
THE INFLUENCE OF SPATIAL FAMILIARITY ON THE LANDMARK SALIENCE SENSIBILITY IN PEDESTRIAN NAVIGATION ENVIRONMENT
}

\author{
Xiao Li a, b, Xin-Qian Wu, ${ }^{a}$, Zi-He Yin a, b, Jie Shen a, b, c, * \\ ${ }^{a}$ Key Laboratory of Virtual Geographic Environment (Nanjing Normal University), Ministry of Education, Nanjing \\ 210023, China - (shenjie, lixiao, wuxinqian, yinzihe)@ njnu.edu.cn \\ ${ }^{\mathrm{b}}$ School of Geographic Science, Nanjing Normal University, No.1, Wenyuan Road, Xianlin District, Nanjing, China \\ c Jiangsu Center for Collaborative Innovation in Geographical Information Resource Development and Application, \\ Nanjing, China
}

Commission IV, WG IV/4

KEY WORDS: Spatial Familiarity, Landmark Salience Sensibility, Pedestrian Navigation, Landmark Visual Salience, Landmark Semantic Salience, Landmark Structural Salience

\begin{abstract}
:
To contribute to a more effective design of landmark navigation guidance, this paper is concerned with the relationship between the spatial familiarity and landmark salience, which includes visual, semantic and structural attributes. The link of those two is the subjective judgment of users, which is called landmark salience sensibility. In order to explore the influence of spatial familiarity on the landmark salience sensibility, we selected two types of experimental area including campus and commercial district and four groups of experimental subject with different spatial familiarity degree. After the whole walking process, subjects are asked to draw a navigation sketch for themselves. Depending on the landmarks remaining in the sketch, we calculated the three attributes of the mean landmark salience to represent the landmark salience sensibility of each group for both paths. The result shows that with the increase of spatial familiarity, the landmark salience sensitivity is gradually reduced and the ascending order of attention degree to the attributes of the landmark salience is visual, semantic and structural salience. This conclusion is supportive to the study of landmark extraction and pedestrian guidance. Because the outdoor landmarks are analysed, we propose that in the future indoor landmarks are needed to be concerned.
\end{abstract}

\section{INTRODUCTION}

Pedestrian navigation has been more and more popular in recent years, and it requires very detailed information on the surrounding environment with high-precision positioning. In order to apply to different navigation scenarios and the lower speed of movement (Millonig and Schechtner, 2007a), which makes landmarks play a more important role in pedestrian navigation especially when people is in an unfamiliar environment (Lee, Tappe and Klippel, 2002a). Landmarks provide clearer clues to pedestrian navigation relative to distance, direction and road names, helping users to perform identity and path decision tasks easily (Hile, Grzeszczuk and Liu, 2009).

In the pedestrian navigation environment, the prominent visual, semantic and structural attributes of landmarks make surrounding environment a significant contrast so that in this case users do not need extra effort to recognize the landmark because of its own salience. In the process of selection of landmarks in the navigation, based on the priori space knowledge in the mind, people use the landmark salience as input information to complete the information processing process. And the output subjective judgment result is called landmark salience sensibility. With the change of the priori space knowledge of users, the landmark salience sensibility is also changed. The spatial familiarity is the description of the number, quality and scope of spatial knowledge (Golledge, 1999a). In the pedestrian navigation environment, it is also the main measure of the knowledge of the a priori space which must be an important influencing factor of landmark salience sensibility.

This paper will explore the influence of spatial familiarity on the landmark salience sensibility. Respectively, the path in the campus area of Nanjing Normal University and another path in the commercial area around the campus as experimental paths are selected. Four groups of experimental subjects are unfamiliar, generally familiar, well familiar or very familiar with the experimental environment based on their different grade. By evaluating the spatial familiarity and ANOVA of the evaluation results, it was proved that the differences in spatial familiarity between the experimental groups are significant. Then, the experimental subjects are asked to complete the navigation tasks. The visual, semantic and structural salience sensibility of landmarks are measured, and the law of the spatial familiarity influence is summarized.

The present study is organized as follows. Section 2 presents the background and related work. Section 3 introduces the design of the experiment. Section 4 presents the statistics results and the analysis. Section 5 summarizes this article and talks about the future work.

\section{BACKGROUND AND RELATED WORK}

\subsection{Landmark salience and landmark salience sensibility}

Landmark salience is the landmark nature of the landmark itself, the strong contrast with the surrounding environment resulting 
in the attraction to people. Appleyard (1969) summarizes these characteristics as unique form, visibility, function and cultural significance. Sorrows and Hirtle (1999) think that the landmark salience should be divided into three aspects: visual, semantic and structural salience. The visual is the appearance part, the semantic is the historical and cultural part and the structural refers to the prominence of landmarks in spatial structural. The prominence of landmarks takes more emphasis on the subjective judgments of users about landmark attraction. The landmark salience is only the information input, which is the object of the process of information processing, such as perception and cognition in the mind. In addition, the result is the subjective judgment of the landmark, which is the landmark salience sensibility.

At present, the evaluation method of landmark salience is already mature. Raubal and Winter (2002) proposed a calculation model of landmark visual, semantic and structural salience based on the theoretical framework of landmarks proposed by Sorrows and Hirtle (1999) earlier. Nothegger, Winter and Raubal (2004a) modified and tested this model and proved its feasibility. Klippel and Winter (2005a) divided it into multiple types based on the relative positional relationship between the landmark and the path, and proposed a method to calculate the prominence of the landmark structural based on this classification. Other scholars (Haken and Portugali, 2003a; Tezuka and Tanaka, 2005) studied the evaluation methods of salience from the theoretical point of view and data sources. Caduff (2008a) proposed a perceptual, cognitive and context three significant vector of the landmark salience of the evaluation model that uses the Bayesian network to study the causal relationship among the spatial object, the observer and the environment in the model.

\subsection{Spatial familiarity}

Spatial familiarity is a description of the quantity, precision, and completeness of spatial knowledge (Lovelace, Hegarty and Montello, 1999), which is a description of the content, precision, and range of cognitive maps. The increase in spatial familiarity represents the increase in the number, precision and completeness of the individual's knowledge of space in a certain location (Burgmanis, Krisjane and Skilters, 2014a), the accuracy of the position, distance and direction of the objects in different dimensions in space knowledge (Chalmers and Knight, 1985a) and the control degree of the local environment (Beguin and Romero, 1996a). Due to the environmental factors, individual factors and individual and environmental interaction factors, there are individual differences in spatial familiarity (Demirbaş, 2001b). At the same time, because of the consistency of the population in a certain direction of the deviation, spatial familiarity shows the similarity of the population, and the study of spatial familiarity has a rule to follow.

There are two types of evaluation methods of spatial familiarity including measurement and calculation. The measurement method can be divided into the user self-evaluation (Couclelis, Golledge and Gale, 1987a) and user behaviour-based measurement which includes user behaviour included handdrawn sketches and marked landmark locations, directions, and so on (Tversky, 2003a; Montello, Waller and Hegarty, 2004a). The calculation method is based on GPS trajectory data (Meneses and Moreira, 2006; Haeren, 2015a) and the location microblogging (Savage, 2012a; Wang, 2015).

\subsection{Related empirical studies}

The studies of the effect of spatial familiarity on the landmark salience are less. Lovelace and Montello (1999) classified landmarks as decision-making landmarks, potential decisionmaking landmarks and long-distance landmarks based on landmark structural salience, and pointed out that the use of potential decision-making landmarks on the familiar roads is more frequent and decision-making landmarks and longdistance landmarks were used more on unfamiliar roads. Landmark signs are important both in familiar and unfamiliar environments. Savage, Chun and Chavez (2012a) also argued that when the user's spatial familiarity increased, the structural salience of the decision-making landmarks was reduced, and the user was more likely to explore other unfamiliar spatial objects.

\section{EXPERIMENTAL DESIGN}

With the change of spatial familiarity, the change of landmark visual, semantic and structural salience sensibility is studied in the experiment.

\subsection{Experimental paths}

The experiment was taken over two paths, and each path contains five decision points. The experimental path one is part of the campus area of Nanjing Normal University, and the experimental path two is part of commercial area around the campus. The detailed paths are shown as Figure 1.
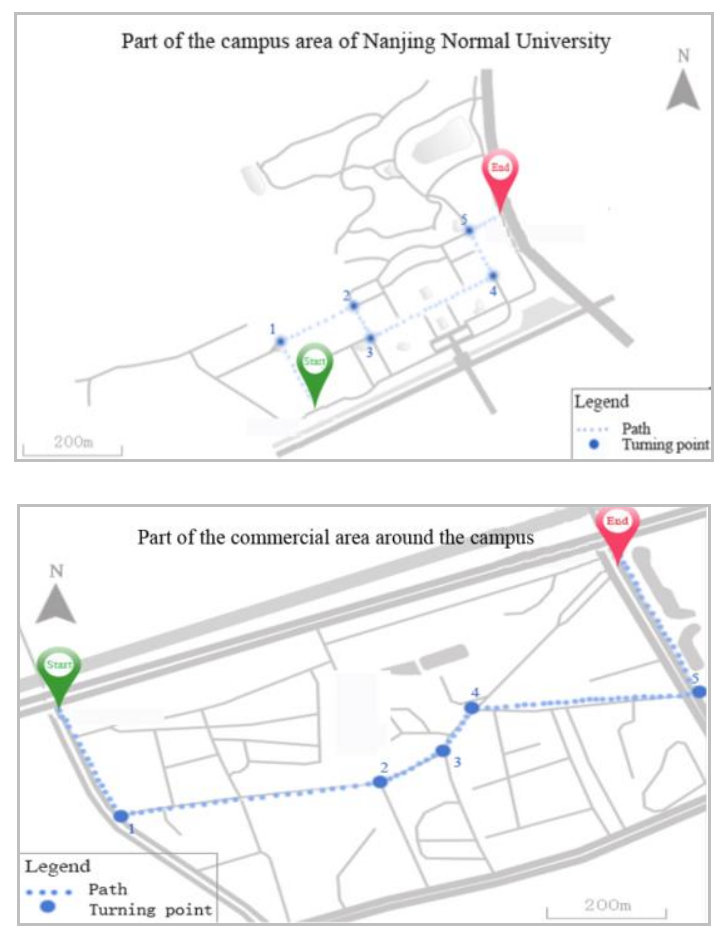

Figure 1. Experimental paths

\subsection{Experimental Subjects}

The total number of experimental subjects is 50. The subjects are required with normal visual or corrected visual acuity and the average age of 20 years (age range: 17 to 24 years old). The subjects who are all college students are divided equally into five groups (A, B, C, D and E) according to the familiarity with the experimental paths, and each group includes equal number of men and women. Group A is subjects outside Nanjing 
Normal University (NNU) and never visiting the experimental area or the experimental area map, and the other groups respectively are freshman, sophomore, junior, senior subjects of NNU. The subjects are shown as Figure 2.

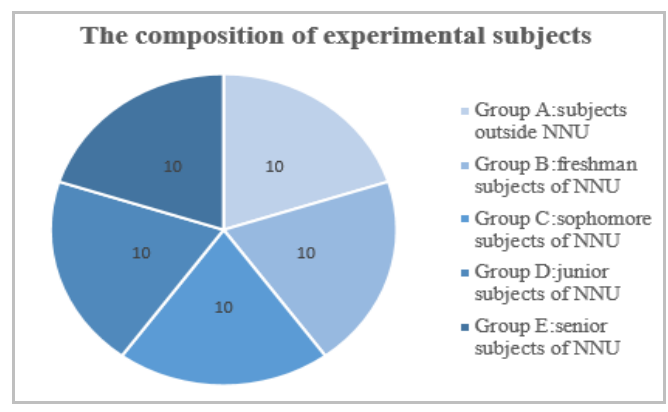

Figure 2. Experimental subjects

\subsection{Experimental procedures}

Before the start of the task, it needs to clear the experimental requirements and specifications in order to reduce the impact of experimental operation proficiency on the experimental results. The subjects receive the training on the experimental operation ability, such as using "ten" mark marking the location in the experimental area sketch and hand-painted experimental path sketch and other operations.

The experiment is divided into three parts, namely, the typical analysis of the experimental path, the typical analysis of the experimental object and the evaluation of the landmark salience. In order to make results of the landmark salience assessment accurate, it is necessary to ensure that the experimental path and the experimental object are typical.

3.3.1 The typical analysis of experimental path: The typicality of the experimental path refers to two aspects. One is that the surrounding landmarks of experimental paths show the landmark visual, semantic and structural salience richly, and the other is that three kinds of landmark saliences need to account for uniformity. The typicality of the experiment refers to the test groups having the significant difference of the spatial familiarity on the experimental area. The typical analysis of the experimental path is done by the evaluation of the salience of the surrounding landmarks and statistical results. The specific measuring methods are based on the research of Raubal and Winter (2002) and Nothegger, Winter and Raubal (2004a).

3.3.2 The typical analysis of experimental object: The typical analysis of the experimental object is achieved by spatial familiarity assessment in the experimental environment. That is, the subjects are required to complete the identification of the landmarks according to the pictures, the identification of the landmarks by name and the determination of landmark position. The target test landmarks are obtained through the public awareness of top ten survey results in the experimental area, and the standard images of 10 landmarks in the experimental area are given in the spatial familiarity assessment.

In the identification of landmarks according to the picture, the test images randomly selected are given. The subjects are required to select the test image in which the landmark corresponds to the same landmark in the standard image. In the identification of landmarks by name, the randomly sorted names to be tested are given. The subjects are asked to select the same landmarks from the standard images according to the names to be tested. In the task of determining the landmark position, the subjects are asked to mark the location of the landmarks in the experimental area sketch which contains only the main road network of the experimental area.

3.3.3 Evaluation of landmark salience : In the task of the landmark salience assessment, the subjects are required to follow the experimenter to conduct the pedestrian tasks of experimental path. When the subjects are in tasks, they need to observe the surrounding environment carefully. After the subjects have completed the pedestrian tasks of experimental path, they will be taken away from the simulated site. In order to be able to complete the task, the subjects need to complete the pedestrian task again after one month accurately, the subjects are asked to draw a navigation sketch for himself. In addition to the sketch, the subjects will not be able to get any help or tips in the navigation process. The drawing task is done on A4 paper and can't be exchanged or accessed during drawing. Finally, the subjects are asked to extract the landmarks contained in their own hand-drawn sketches and sort the landmark visual, semantic and structural salience.

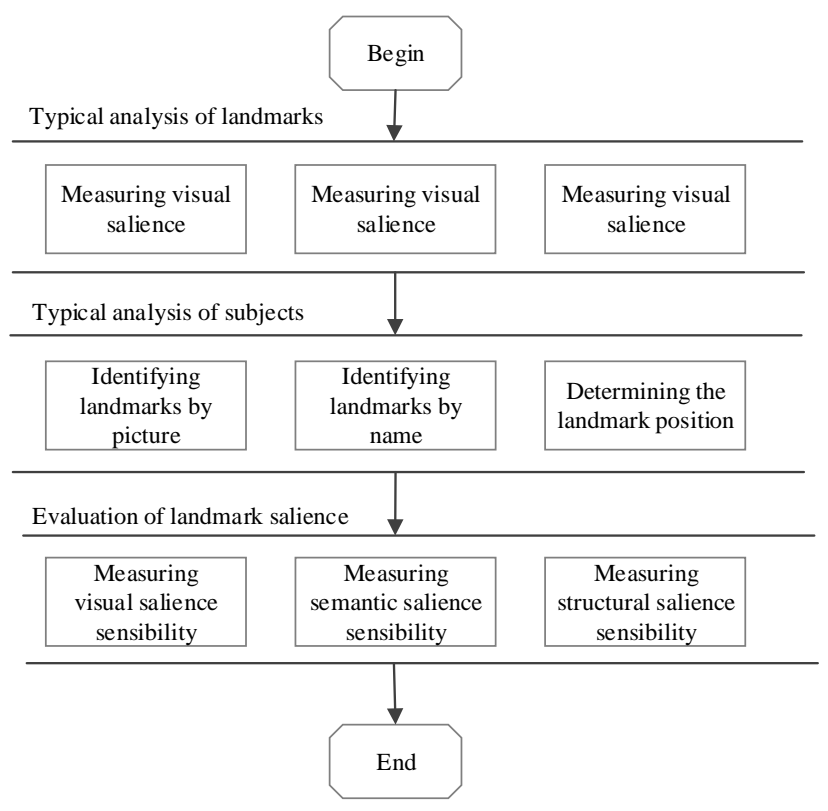

Figure 3. Experimental procedures

\section{EXPERIMENTAL ANALYSIS}

The experiment is completed in January 2016. All subjects are required to complete all experimental tasks as required by the experiment. According to the experimental procedures, the experimental results mainly include three parts, namely, the evaluation results of the landmark salience included in the experimental path, the evaluation results of the experimental object spatial familiarity and the evaluation results of the landmark salience in the sketch.

\subsection{Evaluation of the landmark salience contained in the} experimental path

The visual, semantic and structural salience of the landmarks contained in the experimental area are obtained by referring to the objective evaluation method of landmarks of Raubal and Winter (2002) and Nothegger, Winter and Raubal (2004a). 
Then we calculate the proportion of visual, semantic and structural salience of the landmarks in the two experimental paths. The formula is shown in Equation 1.

$$
\begin{aligned}
& \mathrm{P}_{\mathrm{vis}}=\frac{\sum \mathrm{S}_{\mathrm{vis}}}{\sum \mathrm{S}_{\mathrm{vis}}+\mathrm{S}_{\mathrm{sem}}+\mathrm{S}_{\mathrm{str}}} \times 100 \% \\
& \mathrm{P}_{\mathrm{sem}}=\frac{\sum \mathrm{S}_{\mathrm{sem}}}{\sum \mathrm{S}_{\mathrm{vis}}+\mathrm{S}_{\mathrm{sem}}+\mathrm{S}_{\mathrm{str}}} \times 100 \% \\
& \mathrm{P}_{\mathrm{str}}=\frac{\sum \mathrm{S}_{\mathrm{str}}}{\sum \mathrm{S}_{\mathrm{vis}}+\mathrm{S}_{\mathrm{sem}}+\mathrm{S}_{\mathrm{str}}} \times 100 \%
\end{aligned}
$$

$\mathbf{P}_{\text {vis }}, \mathbf{P}_{\text {sem }}, \mathbf{P}_{\text {str }}$ are respectively the percentage of the proportion of visual salience, semantic salience and structural salience of the landmarks. $\mathrm{S}_{\mathrm{vis}}, \mathrm{S}_{\mathrm{sem}}, \mathrm{S}_{\mathrm{str}}$ are respectively the visual salience, semantic salience and structural salience of the landmarks. The results are shown in Figure 4.

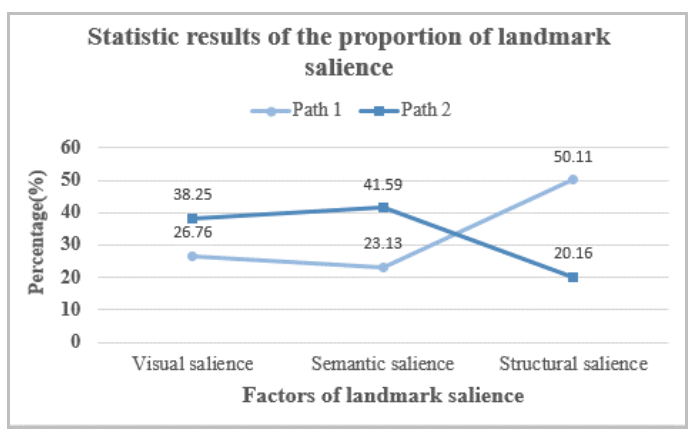

Figure 4. Statistic results of the proportion of landmark salience included experimental path

It can be seen from Figure 4 that the structural salience of experimental path 1 is more pronounced than visual salience and semantic salience. While, compared with the structural salience, the visual salience and semantic salience of experimental path 2 are relatively prominent. The two experimental paths complement each other, showing a certain level of typicality. More details are seen from the Figure 4 as follows:

\section{(1) Lower visual and semantic salience of the landmarks in the experimental path 1}

As the experimental path 1 belongs to the campus area, in which soccer field, basketball court and other landmark height is zero, resulting in having no positive area, shape factors and shape deviation values. Besides, the colour of campus landmarks is single, especially architectural landmarks, reducing the colour eigenvalues. The above reasons lead to a lower visual salience.

As to the semantic salience, the history and culture of other landmarks are less important than that of Jingwen Library, Jingwen Ding and Sports Center. Besides, soccer fields, basketball courts and other landmarks are not external logo, and the functional importance of school bus platform, water room and other landmarks are low in the POI classification. So the above factors lead to a lower percentage of the semantic salience of the landmarks in the experimental path 1.

(2) Higher visual and semantic salience of the landmarks in the experimental path 2

The experimental path 2 locates in commercial blocks, in which landmarks are the majority of shops with obvious external signs, a certain height and the positive area that is rectangular. In addition to KFC, pharmacies and other shops, the experimental path 2 also includes Xianlin Community Hospital, Qixia District Government and other important landmarks. There is a strong functional importance in POI classification at the experimental path 2 , and the landmark colour of commercial landmarks is significantly more colourful than the campus area landmarks'. The above reasons make the percentage of visual and semantic salience of the landmarks higher in the experimental path 2 .

\section{(3) Different in the proportion of structural salience of the landmarks between two paths}

Although the difference of the calculation results of the structural salience is not obvious between the experimental path 1 and the experimental path 2. But, the proportion of the structural salience in the experimental path 2 is reduced because the visual and semantic salience are prominent in the experimental path 2 .

\subsection{Results of experimental object spatial familiarity assessment}

In the identifying the landmarks according to the image, the subjects are asked to observe the standard images of 10 landmarks, and find the image corresponding to the same landmark from the 20 images to be tested and point out the serial number of the landmark in the standard image sequence. Each question is 0.05 points, and the final score of correct number is obtained after the completion of judgments.

In the identification of landmarks by name, the subjects are asked to observe the standard images of 10 landmarks, and then orderly determine the serial number of 20 names to be tested corresponding to the standard image sequence. Each question is 0.05 points, and the final score of correct number is obtained after the completion of judgments.

In the judgment of the landmark position by name, the subjects are asked to mark the position of the landmarks through the "ten" mark in the experimental area sketch. After the mark is completed, the labourer calculates DI (distortion index) of ten landmarks' position marker to be tested according to the calculation method of distortion index of cognitive map created by $\mathrm{Si}$ (2008a). The distortion index is greater, the correctness of the position marker is lower, and the final score of the judgment result is equal to 1 minus the DI.

The scores of the three tasks are combined into the final score of the spatial familiarity measure, shown in Equation 2.

$$
\mathrm{S}_{\text {tol }}=\mathrm{S}_{\text {pic }} \mathrm{W}_{\text {pic }}+\mathrm{S}_{\text {name }} \mathrm{W}_{\text {name }}+\mathrm{S}_{\text {pos }} \mathrm{W}_{\mathrm{pos}}
$$


$S_{\text {pic }}, S_{\text {name }}, S_{\text {pos }}$ are respectively the final score of identifying the landmark according to the picture, identifying the landmark by name and determining the location of the landmark. $\mathrm{W}_{\text {pic }}$, $\mathrm{W}_{\text {name }}, \mathrm{W}_{\text {pos }}$ are respectively the weight of the score of identifying the landmark according to the picture, identifying the landmark by name and determining the location of the landmark. The final results are set them to $1 / 3$. After summarizing and averaging the results of the spatial familiarity measurements for all subjects, the results are as Figure 5.

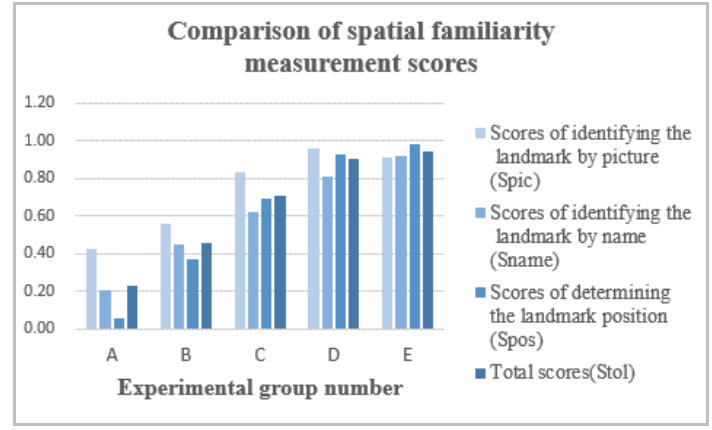

Figure 5. Comparison of spatial familiarity between groups of experimental subjects

Univariate analysis of variance (ANOVA) is used to test the salience of spatial familiarity difference between experimental subjects. The results of analysis of variance (ANOVA) show that the differences in spatial familiarity between the subjects are significant $[\mathrm{F}(4,94)=56.177, \mathrm{P}=0.001]$

In order to confirm whether there is a significant difference in spatial familiarity between the two groups, it is necessary to carry out multiple comparisons. The task is done by using LSD and S-N-K in SPSS. The results of the multiple comparison of the two methods show that there was no significant difference between the 4 and 5 groups (D group and $\mathrm{E}$ group). While there are significant differences in other groups whose sig is less than 0.05 .

The reason for this phenomenon is D, E group of experimental subjects are undergraduate junior, senior students who visit the experimental environment for 2.5 years and 3.5 years. They have a cognitive map of perfect experimental environment in mind, and they are familiar with the experimental environment, so the difference of spatial familiarity between two groups are not obvious. But there are significant differences in spatial familiarity between D, E and other groups. The paper removes group $\mathrm{E}$ which is repeated in order to ensure that the experimental subjects are typical and the grade difference between the experimental subjects is 1 .

\subsection{Analysis on the influence of spatial familiarity on landmark sensitivity}

4.3.1 The results of sensibility of landmark salience assessment: The results of sensibility of landmark salience assessment are mainly from the following two aspects:

(1) The composition of landmark objective salience in handpainted sketches

The paper respectively calculates the mean visual salience of all landmarks $\left(\mathrm{M}_{\mathrm{vis}}\right)$, the mean semantic salience of all landmarks $\left(\mathrm{M}_{\mathrm{sem}}\right)$ and the mean structural salience of all landmarks $\left(\mathrm{M}_{\mathrm{str}}\right)$ in hand-painted sketches painted by the experimental group of subjects. The results are shown in Figure 6.

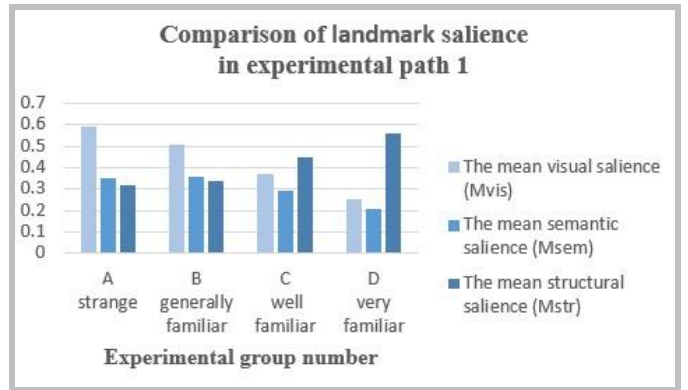

(a) Experimental path 1

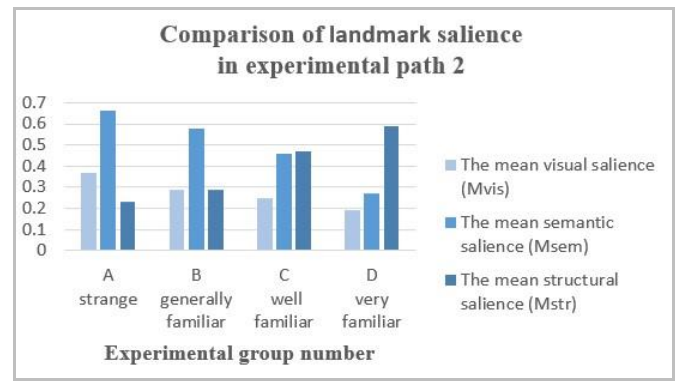

(b) Experimental path 2

Figure 6. Comparison of landmark salience in hand-painted sketches

\section{(2) The ranking of strong degree of landmark salience}

There exist simultaneously visual, semantic and structural salience in landmarks. But spatial familiarity has an impact on it, leading that the attraction of the three landmark saliences for the subjects is not the same. The paper statistics out of the proportion of the landmark salience ranking first from three landmark saliences in the hand-painted sketches according to the ranking of landmark salience for the subjects. The statistical results are shown in Figure 7.

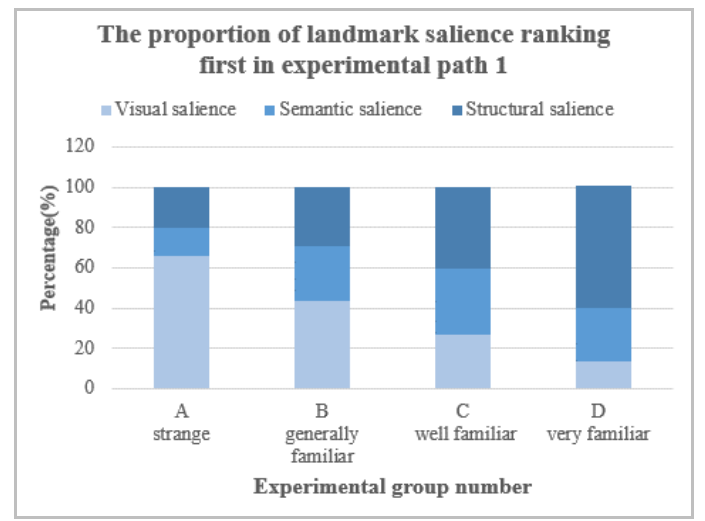

(a) Experimental path 1 


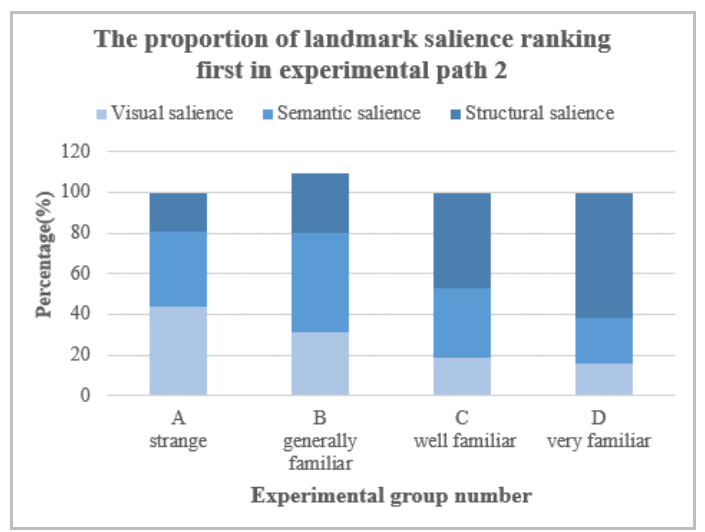

(b) Experimental path 2

Figure 7. The proportion of landmark salience ranking first in the hand-painted sketches painted by subjects

4.3.2 Discussion: According to the statistical results, the effect of spatial familiarity on the landmarks salience is analysed from the following two aspects:

\section{(1) The effect of spatial familiarity on the landmarks objective salience}

From figure 6 , it can be easily observed that there is a great difference among the experimental groups mainly in the following aspects:

First, the visual salience and semantic salience of landmarks are more readily obtained relative to structural salience information. The mean value of the landmarks structural salience is relatively low in the two experimental paths, particularly in the experimental path 1. As shown in Figure 7, the structural salience of the landmarks of experimental path 1 is high, but the salience of the structural salience of the landmarks of group A and group B is still lower than the visual salience and semantic salience.

Second, the composition of landmark salience has a significant impact on the spatial knowledge acquired by unfamiliar users. According to the comparative analysis of the objective salience of the two experimental paths, the visual salience of the experimental path 1 is relatively high, and the semantic salience of the experimental path 2 is relatively high. This makes the visual salience of the landmarks in the group $\mathrm{A}$ in the path 1 is significantly high and the structural salience in path 2 is high.

Third, the higher the spatial familiarity, the more emphasis on the structure of landmarks. It can be directly observed from the figure that with the increase of spatial familiarity, the visual and semantic salience of the two experimental paths are declining continuously, but the structural salience is more and more significant. The reason for this phenomenon is that subjects need to use non-decision-making landmarks to confirm their own in the correct path initially when they lack the knowledge of the environment. However, with more and more familiar with the environment, subjects in the navigation process only need key decision points to remember the navigation path, so the landmark average salience of the structure continued to increase.

Fourth, group with the general familiarity has an increase attention to the landmark which salience is low. On the one hand, the number of landmarks in each experiment is the largest. On the other hand, the mean value of the three factors of group $\mathrm{B}$ are relatively low of group A in both path. The reason is that the spatial familiarity of group B is higher than that of group A. When group B subjects are successful in recognizing landmarks with high objective salience, they begin to shift their attention to other low salience landmarks, resulting in lower means. This phenomenon is consistent with the results of structural salience analysis (Lovelace 1999). Users has more interest in potential decision-making landmarks when they are familiar with decision-making landmarks.

\section{(2) The effect of spatial familiarity on the ranking of the factors of the landmark salience sensibility}

From Figure 7, it can be seen intuitively that the salience sensibility of landmarks changes with the spatial familiarity. In group A, unfamiliar subjects agree that the visual salience of landmarks is more intense, and group B is generally familiar with the semantic salience because of the increases. Group C and group D more likely to put structural salience to the first because of the more intense feelings. In view of the above, in the experimental area, the subjects in the process of exposure to the environment through the process, with the increase in spatial familiarity, the importance of the landmark salience has an ascending order of visual salience, semantic salience and structural salience.

\section{CONCLUSIONS}

In recent years, with the increase number of pedestrian navigation users, the route guidance based on landmarks has been gained more and more attention. The affection of the spatial familiarity to the design of walking navigation has gradually become an important factor of research. The landmark salience sensibility is the subjective judgment of the users' subject based on their prior space knowledge for the landmark. It is influenced by the spatial familiarity, and the influence law can be applied to the extraction of the landmarks. For the landmarks based on the user's cognition of the pedestrian navigation guide is also of great significance.

This paper studies the influence law of the spatial familiarity of the user's subject on the landmark salience sensibility including visual, semantic and structural attributes. The typicality of the experimental path is proved by assessing the salience including visual, semantic and structural of all landmarks. By analysing the spatial familiarity of the experimental subjects and the variance analysis of the significant differences in the spatial familiarity among the experimental groups, the filter of the experimental subjects was completed, and the typicality of the experimental objects was ensured. Based on this, the experimental object completed the evaluation of the apparent sensibility of the surrounding landmarks. The experimental results show that when the degree of spatial familiarity is low, the proportion of the sensibility of the three factors of the measured landmarks is consistent with the salience of the landmarks. With the increase of spatial familiarity, the ascending order of attention to the landmark salience is visual, semantic and structural salience.

In the future work, we will apply this law to the landmark extraction, enriching the landmark-based pedestrian navigation path guide generation method. In addition, the spatial familiarity for the significant impact of indoor landmarks will be concerned. 


\section{ACKNOWLEDGEMENTS}

The research work presented in this paper is supported by NSFC (National Natural Science Foundation of China) project No. 41371433, The National Key Research and Development Program of China, Intergovernmental international cooperation in science and technology innovation Project of China and Czech (2016YFE0131600).

\section{REFERENCES}

Appleyard, D., 1969. Why buildings are known -- a predictive tool for architects and planners. In: Environment and Behavior, Vol. 1, pp. 131-156.

Beguin, H., Romero, V. L., 1996a. Individual cognition of urban neighbourhoods over space and time: a case study. Environment and Planning A, 28(4), pp. 687-708.

Burgmanis, G̦., Krišjāne, Z., Šḳilters, J., 2014a. Acquisition of spatial knowledge in different urban areas: evidence from a survey analysis of adolescents. Cognitive processing, 15(3), pp. 373-383.

Caduff, D., Timpf, S., 2005. The Landmark Spider: Weaving the Landmark Web. In: 5th Swiss Transport Research Conference, Switzerland, pp. 619-625.

Chalmers, D. J., Knight, R. G., 1985a. The Reliability of Ratings of the Familiarity of Environmental Stimuli a Generalizability Analysis. Environment and Behavior, 17(2), pp. 223-238.

Couclelis, H., Golledge, R. G., Gale, N., et al., 1987a. Exploring the anchor-point hypothesis of spatial cognition. Journal of Environmental Psychology, 7(2), pp. 99-122.

Demirbaş, G. U. D., 2001b. Spatial familiarity as a dimension of wayfing. Bilkent university.

Golledge, R. G., 1999a. Human wayfinding and cognitive maps. Wayfinding behavior: Cognitive mapping and other spatial processes, pp. 5-45.

Haken, H., Portugali, J., 2003a. The face of the city is its information. Journal of Environmental Psychology, 23(4), pp. 385-408.

Hile, H., Grzeszczuk, R., Liu, A., et al., 2009. Landmark-based pedestrian navigation with enhanced spatial reasoning. In: Pervasive Computing. Pervasive, Lecture Notes in Computer Science, Berlin Heidelberg, Vol. 5538, pp. 59-76.

Klippel, A., Winter, S., 2005a. Structural salience of landmarks for route directions. Lecture notes in computer science, pp. 347362.

Lee, P., Tappe, H., Klippel, A., 2002a. Acquisition of landmark knowledge from static and dynamic presentation of route maps. KI, 16(4), pp. 32-34.

Lovelace, K. L., Hegarty, M., Montello, D. R., 1999. Elements of good route directions in familiar and unfamiliar environments. In: Spatial information theory, Cognitive and computational foundations of geographic information science. Springer Berlin Heidelberg, pp. 65-82.
Millonig, A., Schechtner, K., 2007a. Developing landmarkbased pedestrian-navigation systems. Intelligent Transportation Systems, IEEE Transactions on Intelligent Transportation Systems, 8(1), pp. 43-49.

Meneses, F., Moreira, A., 2006. Using GSM CellID positioning for place discovering. In: Pervasive Health Conference and Workshops, Berlin Heidelberg, pp. 1-8.

Montello, D. R., Waller, D., Hegarty, M., et al., 2004a. Spatial memory of real environments, virtual environments, and maps. Atherosclerosis, 41(2-3), pp. 185-192.

Nothegger, C., Winter, S., Raubal, M., 2004a. Selection of salient features for route directions. Spatial cognition and computation, 4(2), pp. 113-136.

Raubal, M., Winter, S., 2002. Enriching wayfinding instructions with local landmarks. In: International conference on geographic information science. Springer-Verlag, Vol. 2478, pp. 243-259.

Savage, N. S., Chun, W., Chavez, N. E., et al., 2012a. Seems Familiar: An Algorithm for Inferring Spatial Familiarity Automatically. Cs.ucsb.edu.

Si, S., Xue L., Yu, L., 2008a. Beijing Residents' Cognitive Map Distortion and Sources Analysis Based on Sketch Mapping Method. Journal of Geographical Sciences, 63(6), pp. 625-634.

Sorrows, M. E., Hirtle, S. C., 1999. The nature of landmarks for real and electronic spaces. In: Spatial information theory. Cognitive and computational foundations of geographic information science, Springer Berlin Heidelberg, pp. 37-50.

Tezuka, T., Tanaka, K., 2005. Landmark extraction: A web mining approach. In: Spatial information theory, Springer Berlin Heidelberg, pp. 379-396.

Tversky, B., 2003a. Navigating by mind and by body. Lecture notes in computer science, pp. 1-10.

Van Haeren, M., Mackaness, W., 2015a. The Influence of familiarity on route choice: edinburgh as a case study.

Wang, W., 2015. Using Location-Based Social Media for Ranking Individual Familiarity with Places: A Case Study with Foursquare Check-in Data. In: Progress in Location-Based Services. Springer International Publishing, pp. 171-183. 